\title{
ON THE SPECIFIC AREA OF INHOMOGENEOUS BOOLEAN MODELS. EXISTENCE RESULTS AND APPLICATIONS
}

\author{
ELENA ViLLA \\ Dept. of Mathematics, University of Milan, Via Saldini 50, 20133, Milano, Italy \\ e-mail: elena.villa@unimi.it \\ (Accepted April 7, 2010)
}

\begin{abstract}
The problem of the evaluation of the so-called specific area of a random closed set, in connection with its mean boundary measure, is mentioned in the classical book by Matheron on random closed sets (Matheron, 1975, p. 50); it is still an open problem, in general. We offer here an overview of some recent results concerning the existence of the specific area of inhomogeneous Boolean models, unifying results from geometric measure theory and from stochastic geometry. A discussion of possible applications to image analysis concerning the estimation of the mean surface density of random closed sets, and, in particular, to material science concerning birth-and-growth processes, is also provided.
\end{abstract}

Keywords: geometric measure theory, mean surface density, outer Minkowski content, specific area, stochastic geometry.

\section{INTRODUCTION}

In this paper we offer an overview of some recent results concerning the existence of the specific area of inhomogeneous Boolean models, and its relationship with the mean surface density of the involved random sets. Applications to birth-andgrowth stochastic processes are also discussed.

If a $n$-dimensional random closed set $\Theta$ in $\mathbb{R}^{d}$ is such that $\mathbb{E}\left[\mathscr{H}_{\Theta}^{n}\right]$ is absolutely continuous with respect to $\mathscr{H}^{d}$, where $\mathscr{H}^{n}$ is the $n$-dimensional Hausdorff measure, and $\mathscr{H}_{\Theta}^{n}$ is its restriction to $\Theta$, then the density (or Radon-Nikodym derivative) of $\mathbb{E}\left[\mathscr{H}_{\mid \Theta}^{n}\right]$ with respect to $\mathscr{H}^{d}$ is called mean density of $\Theta$. Whenever the mean density of $\Theta$ exists, we denote it by $\lambda_{\Theta}$.

The problem of the evaluation of the mean densities of lower dimensional random closed sets, and in particular of the mean surface density, is of interest in several real applications.

In Ambrosio et al. (2009) the notion of local mean n-dimensional Minkowski content of a random closed set has been introduced in order to provide approximations of the mean density of $n$-dimensional random closed sets in $\mathbb{R}^{d}$. With regard to the approximation of the mean surface density of a $d$ dimensional random closed set, the concept of specific area introduced in Matheron (1975, p. 50) turns out to be closely related to the notion of local mean outer Minkowski content of a random closed set. We remind that, given a random closed set $\Theta$ in $\mathbb{R}^{d}$, the specific area $\sigma_{\Theta}(x)$ of $\Theta$ at a point $x$ in $\mathbb{R}^{d}$ is defined as the following limit

$$
\sigma_{\Theta}(x):=\lim _{r \downarrow 0} \frac{\mathbb{P}\left(x \in \Theta_{\oplus r} \backslash \Theta\right)}{r},
$$

whenever it exists. $\left(\Theta_{\oplus r}\right.$ denotes here the parallel set of $\Theta$ at distance $r$, i.e., $\Theta_{\oplus r}:=\left\{x \in \mathbb{R}^{d}: \operatorname{dist}(x, \Theta) \leq r\right\}$.) It is mentioned in Matheron (1975) that the definition of $\sigma_{\Theta}(x)$ is the "translation" into probabilistic terms of the following limit

$$
\lim _{r \downarrow 0} \frac{\mathscr{H}^{d}\left(K_{\oplus r} \backslash K\right)}{r}, \quad K \subset \mathbb{R}^{d} \text { compact. }
$$

The limit above, whenever it exists finite, is called area of $K$ in Matheron (1975), or outer Minkowski content of $K$ in Ambrosio et al. (2008). As right derivative at $r=0$ of the volume function $V(r):=$ $\mathscr{H}^{d}\left(K_{\oplus r}\right)$, it is intuitively clear that there exist compact subsets of $\mathbb{R}^{d}$ such that the limit in Eq. 2 equals the surface measure $\mathscr{H}^{d-1}(\partial K)$ of $K($ e.g., if $K$ is convex with nonempty interior), that explains the name area of $K$; actually, examples of subsets of $\mathbb{R}^{d}$, even closure of their interior, such that the above limit differs from the $\mathscr{H}^{d-1}$-measure of their boundary can be provided. Notice that a computer graphics representation of lower dimensional sets in $\mathbb{R}^{2}$ is anyway provided in terms of pixels, which can offer only a 2-D box approximation of points in $\mathbb{R}^{2}$ (an interesting discussion on this is contained in Kärkkäinen et al., 2002); therefore, the possibility of evaluating and estimating the surface measure of a set (the mean surface density for random sets) by the 
volume measure of the Minkowski enlargement of the involved set by Eq. 2 (by Eq. 1 in the stochastic case) might be a solution to problems of this kind.

\section{SPECIFIC AREA AND LOCAL MEAN OUTER MINKOWSKI CONTENT}

Throughout the paper $\mathscr{H}^{n}$ is the $n$-dimensional Hausdorff measure, $\mathrm{d} x$ stands for $\mathscr{H}^{d}(\mathrm{~d} x), \mathscr{B}_{\mathbb{R}^{d}}$ is the Borel $\sigma$-algebra of $\mathbb{R}^{d}$ and $\mathscr{H}_{\mid A}^{n}$ denotes the restriction of $\mathscr{H}^{n}$ to a $\mathscr{H}^{n}$-measurable set $A \subset \mathbb{R}^{d}$ (i.e., $\mathscr{H}_{\mid A}^{n}(B)=$ $\mathscr{H}^{n}(A \cap B)$ for all $\left.B \in \mathscr{B}_{\mathbb{R}^{d}}\right)$. $B_{r}(x)$ will denote the closed ball with centre $x$ and radius $r$, whereas for any integer $n$ we denote by $b_{n}$ the volume of the unit ball in $\mathbb{R}^{n}$.

We recall that, given a subset $S$ of $\mathbb{R}^{d}$ and an integer $n$ with $0 \leq n \leq d$, the $n$-dimensional Minkowski content of $S$ is defined by

$$
\mathscr{M}^{n}(S):=\lim _{r \downarrow 0} \frac{\mathscr{H}^{d}\left(S_{\oplus r}\right)}{b_{d-n} r^{d-n}}
$$

whenever the limit exists finite.

Let $A \in \mathscr{B}_{R^{d}}$; the quantity $\mathscr{S} \mathscr{M}(A)$ defined as (Ambrosio et al., 2008)

$$
\mathscr{S} \mathscr{M}(A):=\lim _{r \downarrow 0} \frac{\mathscr{H}^{d}\left(A_{\oplus r} \backslash A\right)}{r},
$$

provided that the limit exists finite, is called outer Minkowski content of $A$. Note that if $A$ is lower dimensional, then $\mathscr{S} \mathscr{M}(A)=2 \mathscr{M}^{d-1}(A)$, whereas if $A$ is a $d$-dimensional set, closure of its interior, then $A_{\oplus r} \backslash$ $A$ coincides with the outer Minkowski enlargement at distance $r$ of $\partial A$.

In this section we show why the specific area can be interpreted as the translation into probabilistic terms of the outer Minkowski content. For basic definitions and results of stochastic geometry we refer to Stoyan et al. (1995), Baddeley et al. (2007), and Schneider and Weil (2008).

If the limit

$$
\lim _{r \downarrow 0} \frac{\mathscr{H}^{d}\left(\left(A_{\oplus r} \backslash A\right) \cap B\right)}{r}
$$

exists finite for any $B \in \mathscr{B}_{\mathbb{R}^{d}}$ such that $\mathscr{H}^{d-1}(\partial A \cap$ $\partial B)=0$, then we say that $A$ admits local outer Minkowski content. Now, let us consider a random closed set $\Theta$ in $\mathbb{R}^{d}$, that is a measurable map $\Theta$ :
$(\Omega, \mathfrak{F}, \mathbb{P}) \rightarrow\left(\mathbb{F}, \sigma_{\mathbb{F}}\right)\left(\mathbb{F}\right.$ and $\sigma_{\mathbb{F}}$ denote here the class of the closed subsets in $\mathbb{R}^{d}$ and the $\sigma$-algebra generated by the so-called hit-or-miss topology (Matheron, 1975), respectively). If the limit

$$
\lim _{r \downarrow 0} \frac{\mathbb{E}\left[\mathscr{H}^{d}\left(\left(\Theta_{\oplus r} \backslash \Theta\right) \cap B\right)\right]}{r}
$$

exists finite for any $B \in \mathscr{B}_{\mathbb{R}^{d}}$ such that $\mathbb{E}\left[\mathscr{H}^{d-1}(\partial \Theta \cap\right.$ $\partial B)]=0$, then we say that $\Theta$ admits local mean outer Minkowski content. Let us notice that, by a straightforward application of Fubini's theorem, Eq. 3 can be written equivalently

$$
\lim _{r \downarrow 0} \int_{B} \frac{\mathbb{P}\left(x \in \Theta_{\oplus r} \backslash \Theta\right)}{r} \mathrm{~d} x,
$$

if furthermore $\Theta$ is stationary (and so $\mathbb{P}\left(x \in \Theta_{\oplus r} \backslash \Theta\right.$ ) is constant), then, by choosing $B=[0,1]^{d}$ in Eq. 4 , we have that the specific area $\sigma_{\Theta}$ is constant, given by

$$
\sigma_{\Theta}=\lim _{r \downarrow 0} \frac{\mathbb{E}\left[\mathscr{H}^{d}\left(\left(\Theta_{\oplus r} \backslash \Theta\right) \cap[0,1]^{d}\right)\right]}{r} .
$$

More in general, as we shall see in the next sections, if the boundary $\partial A$ of a $d$-dimensional Borel set $A \in \mathbb{R}^{d}$ is "sufficiently regular", then $\mathscr{S} \mathscr{M}(A)=$ $\mathscr{H}^{d-1}(\partial A)$; thus, it is intuitive that for "sufficiently regular" random closed sets we may have

$$
\mathbb{E}\left[\mathscr{H}^{d-1}(\partial \Theta \cap B)\right]=\lim _{r \downarrow 0} \frac{\mathbb{E}\left[\mathscr{H}^{d}\left(\left(\Theta_{\oplus r} \backslash \Theta\right) \cap B\right)\right]}{r},
$$

and

$$
\lim _{r \downarrow 0} \int_{B} \frac{\mathbb{P}\left(x \in \Theta_{\oplus r \backslash \Theta}\right) \mathrm{d} x}{r}=\int_{B} \sigma_{\Theta}(x) \mathrm{d} x,
$$

so that $\sigma_{\Theta}(x)$ turns out to be the mean surface density of $\Theta$. This is true for instance when $\Theta$ satisfies a (local) Steiner formula; in this case the limit in Eq. 5 can be studied in terms of the quermass densities (or Minkowski functionals) associated to $\Theta$, and so by means of tools from integral geometry mainly (Hug, 2000; Weil, 2001; Hug et al., 2006; Baddeley et al., 2007 , and references therein). For other related works see also Hug and Last (2000), Hug et al. (2004), and Kiderlen and Rataj (2006).

The passage from stationary to nonstationary random closed sets, and from convex to more general grains gives rise to nontrivial problems. We will focus here on the specific area of inhomogeneous Boolean models; for this kind of random closed sets (widely used in real applications in Material Science, as we shall mention in the last section) the relationship between specific area and outer Minkowski content (of the typical grain) is more evident. Let us see why briefly. 
Let $\Xi$ be a Boolean model in $\mathbb{R}^{d}$ (Stoyan et al., 1995; Baddeley et al., 2007; Schneider and Weil, 2008) whose typical grain $Z_{0}$ has topological boundary $\partial Z_{0}$ with Hausdorff dimension $d-1 \mathbb{P}$-a.s. It is well known that Boolean models in $\mathbb{R}^{d}$ can be described by marked Poisson point processes on $\mathbb{R}^{d}$ with marks in the space of centred compact sets. In many examples and applications $Z_{0}$ is uniquely determined by a random quantity, say $S$, in a suitable mark space $\mathbf{K}$; for instance, in the very simple case of random balls, $\mathbb{K}=\mathbb{R}_{+}$and $S$ is the radius, whereas in applications to birth-and-growth processes, in some models $\mathbb{K}=\mathbb{R}^{d}$ and $S$ is the spatial location of the nucleus, in other models $\mathbb{K}=\mathbb{R}_{+}$and $S$ is the birth time of the nucleus (see last section). Therefore, we shall consider (inhomogeneous) Boolean models of the type

$$
\Xi(\omega)=\bigcup_{\left(x_{i}, s_{i}\right) \in \Psi(\omega)} x_{i}+Z_{0}\left(s_{i}\right),
$$

where $Z_{0}(s)$ is a compact subset of $\mathbb{R}^{d}$ containing the origin for any $s \in \mathbf{K}$, and $\Psi$ is the marked Poisson point process in $\mathbb{R}^{d}$ with marks in $\mathbf{K}$ associated to $\Xi$, with intensity measure

$$
\Lambda(\mathrm{d}(x, s))=f(x) \mathrm{d} x Q(\mathrm{~d} s) .
$$

The function $f$ and the probability measure $Q$ on $\mathbf{K}$ are called intensity of $\Xi$ and mark distribution, respectively, and they are commonly assumed to be such that the mean number of grains hitting any compact subset of $\mathbb{R}^{d}$ is finite:

$$
\int_{\mathbf{K}} \int_{\left(-Z_{0}(s)\right)_{\oplus R}} f(x) \mathrm{d} x Q(\mathrm{~d} s)<\infty \quad \forall R>0 .
$$

(For basic definitions and results on the theory of point processes, we refer to Baddeley et al. (2007) and references therein.)

We shall denote by $\operatorname{diam}\left(Z_{0}\right)$ the (random) diameter of $Z_{0}$, and by $\operatorname{disc} f$ the set of all the points of discontinuity of $f$.

From now on let $Z^{x}:=x-Z_{0} \forall x \in \mathbb{R}^{d}$. By the explicit expression of the capacity functional of a Boolean model (Matheron, 1975), it is not difficult to get (Villa, 2010, Eq. 3.1) that

$$
\begin{array}{r}
\sigma_{\Theta}(x):=\lim _{r \downarrow 0} \frac{\mathbb{P}\left(x \in \Xi_{\oplus r} \backslash \Xi\right)}{r}=e^{-\mathbb{E}_{Q}\left[\int_{Z^{x}} f(y) \mathrm{d} y\right] .} \\
\cdot \lim _{r \downarrow 0} \frac{\left(1-\exp \left\{-\mathbb{E}_{Q}\left[\int_{Z_{\oplus r}^{x} \backslash Z^{x}} f(y) \mathrm{d} y\right]\right\}\right)}{r},
\end{array}
$$

where $\mathbb{E}_{Q}$ denotes the expectation with respect to $Q$; thus, in order to obtain sufficient conditions for the existence of $\sigma_{\Theta}(x)$, existence results for limits of the type

$$
\lim _{r \downarrow 0} \frac{1}{r} \int_{A_{\oplus r} \backslash A} f(x) \mathrm{d} x
$$

have to be studied.

Note that in the particular case in which $f$ is constant the limit above exists if and only if $A$ admits outer Minkowski content.

In the next section we recall some recent results on the existence of the outer Minkowski content of Borel subsets of $\mathbb{R}^{d}$, which will be the starting point to obtain existence results for the specific area of random closed sets.

\section{EXISTENCE RESULTS FOR THE OUTER MINKOWSKI CONTENT}

We remind that a compact set $A \subset \mathbb{R}^{d}$ is called $n$ rectifiable $(0 \leq n \leq d-1$ integer $)$ if it is representable as the image of a compact subset of $\mathbb{R}^{n}$ by a Lipschitz map from $\mathbb{R}^{n}$ to $\mathbb{R}^{d}$; more in general, a closed subset $A$ of $\mathbb{R}^{d}$ is called to be countably $\mathscr{H}^{n}$-rectifiable if there exist countably many Lipschitz maps $g_{i}: \mathbb{R}^{n} \rightarrow$ $\mathbb{R}^{d}$ such that $A \backslash \bigcup_{i} g_{i}\left(\mathbb{R}^{n}\right)$ is $\mathscr{H}^{n}$-negligible. (For definitions and basic properties of Hausdorff measure and rectifiable sets see, e.g., Federer, 1969, Falconer, 1985, and Ambrosio et al., 2000.) We call Radon measure in $\mathbb{R}^{d}$ any nonnegative and $\sigma$-additive set function $\mu$ defined on $\mathscr{B}_{\mathbb{R}^{d}}$ which is finite on bounded sets.

Federer's theorem (Federer, 1969, p. 275) on the existence of the $n$-dimensional Minkowski content of $n$-rectifiable compact sets is well known. We recall a generalization of such theorem, proved in Ambrosio et al. (2000, p. 110), to countably $\mathscr{H}^{n}$ rectifiable compact sets and then we shall see that if the boundary of a Borel subset of $\mathbb{R}^{d}$ satisfies similar conditions, then it admits outer Minkowski content.

Theorem 1 Let $A \subset \mathbb{R}^{d}$ be a countably $\mathscr{H}^{n}$-rectifiable compact set and assume that

$$
\eta\left(B_{r}(x)\right) \geq \gamma r^{n} \quad \forall x \in A, \forall r \in(0,1)
$$

holds for some $\gamma>0$ and some Radon measure $\eta$ in $\mathbb{R}^{d}$ absolutely continuous with respect to $\mathscr{H}^{n}$. Then $\mathscr{M}^{n}(A)=\mathscr{H}^{n}(A)$.

Condition (Eq. 9) is a kind of quantitative nondegeneracy condition which prevents $A$ from being too sparse; simple examples (Ambrosio et al., 2000; 2008) show that $\mathscr{M}^{n}(A)$ can be infinite, and $\mathscr{H}^{n}(A)$ arbitrarily small, when this condition fails.

It is reasonable to conjecture that, if the boundary $\partial A$ of a subset $A$ of $\mathbb{R}^{d}$ satisfies the assumptions of 
the theorem above, then $A$ admits outer Minkowski content; in Villa (2009a) it has been proved that the value of $\mathscr{S} \mathscr{M}(A)$ depends on the density of $A$ at its boundary points. We remind that the $d$ dimensional density (briefly, density) of $A$ is defined by Ambrosio et al. (2000)

$$
\theta_{d}(A, x):=\lim _{r \downarrow 0} \frac{\mathscr{H}^{d}\left(A \cap B_{r}(x)\right)}{\mathscr{H}^{d}\left(B_{r}(x)\right)},
$$

provided that the limit exists. It is clear that $\theta_{d}(A, x)$ equals 1 for all $x$ in the interior of $A$, and 0 for all $x$ into the interior of the complement set of $A$, whereas different values can be attained at its boundary points. It is well known (cf. Ambrosio et al., 2000, Theorem 3.61) that if $\mathscr{H}^{d-1}(\partial A)<\infty$, then $A$ has density either 0 or 1 or $1 / 2$ at $\mathscr{H}^{d-1}$-almost every point of its boundary. For every $t \in[0,1]$ and every $\mathscr{H}^{d}$ measurable set $A \subset \mathbb{R}^{d}$ let

$$
A^{t}:=\left\{x \in \mathbb{R}^{d}: \theta_{d}(A, x)=t\right\} .
$$

Intuitively, a small neighborhood of a point $x \in A^{1} \cap \partial A$ is "almost all contained" in $A$, so that it gives no contribution to the volume of $A_{\oplus r} \backslash A$; thus, roughly speaking, we may say that $x$ has negligible weight in the computing of the outer Minkowski content of $A$. Conversely, if $A$ has null density in $x \in \partial A$, then, in a small neighborhood of $x, A_{\oplus r} \backslash A$ "almost all coincides" with the Minkowski enlargement of $\partial A$, so that, roughly speaking, we may say that the weight of $x$ in the computing of the outer Minkowski content of $A$ is twice the weight of a point $y \in A^{1 / 2}$.

In Villa (2009a) the following class of sets (whose boundary satisfies the assumptions of Theorem 1) has been introduced:

Definition 2 (The class $\mathscr{O}$ ) Let $\mathscr{O}$ be the class of Borel sets $A$ of $\mathbb{R}^{d}$ with countably $\mathscr{H}^{d-1}$-rectifiable and bounded topological boundary, such that

$$
\eta\left(B_{r}(x)\right) \geq \gamma r^{d-1} \quad \forall x \in \partial A, \forall r \in(0,1)
$$

holds for some $\gamma>0$ and some probability measure $\eta$ in $\mathbb{R}^{d}$ absolutely continuous with respect to $\mathscr{H}^{d-1}$.

Theorem 3 (Villa, 2009a) The class $\mathscr{O}$ is stable under finite unions and any $A \in \mathscr{O}$ admits outer Minkowski content, given by

$$
\mathscr{S} \mathscr{M}(A)=\mathscr{H}^{d-1}\left(A^{1 / 2}\right)+2 \mathscr{H}^{d-1}\left(\partial A \cap A^{0}\right) .
$$

Remark 4 The set of points

$$
\partial^{*} A:=\mathbb{R}^{d} \backslash\left(A^{0} \cup A^{1}\right)
$$

where the density of $A$ is neither 0 nor 1 is called essential boundary of $A$. It is proved ( $c f$. Ambrosio et al., 2000) that all the sets $A^{t}$ are Borel sets, and that

$$
\mathscr{H}^{d-1}\left(\partial^{*} A \cap B\right)=\mathscr{H}^{d-1}\left(A^{1 / 2} \cap B\right)
$$

for all $B \in \mathscr{B}_{\mathbb{R}^{d}}$.

Remark 5 (The class $\mathscr{O}^{\prime}$ ) In Villa (2009a) it is also proved that the same conclusions of the above theorem hold for a class of Borel subsets of $\mathbb{R}^{d}$, named $\mathscr{O}^{\prime}$, defined similarly to $\mathscr{O}$ by replacing the condition of absolutely continuity of $\eta$ with the assumption that $\mathscr{M}^{d-1}(\partial A)=\mathscr{H}^{d-1}(\partial A)$; then it follows that this class of sets contains all Borel sets with $(d-1)$-rectifiable boundary (and so finite unions of sets with positive reach or with Lipschitz boundary, in particular).

We conclude this section by the following theorem proved in Villa (2010), which generalizes Theorem 3 providing sufficient conditions for the existence of the limit in Eq. 8.

Theorem 6 Let $\mu$ be a positive measure in $\mathbb{R}^{d}$ absolutely continuous with respect to $\mathscr{H}^{d}$ with locally bounded density $f$. Let $A$ belong to $\mathscr{O}$ (or $\left.\mathscr{O}^{\prime}\right)$. If $\mathscr{H}^{d-1}(\operatorname{disc} f)=0$, then

$$
\begin{aligned}
& \lim _{r \downarrow 0} \frac{\mu\left(A_{\oplus r} \backslash A\right)}{r} \\
= & \int_{\partial^{*} A} f(x) \mathscr{H}^{d-1}(\mathrm{~d} x)+2 \int_{\partial A \cap A^{0}} f(x) \mathscr{H}^{d-1}(\mathrm{~d} x) .
\end{aligned}
$$

\section{EXISTENCE RESULTS FOR THE SPECIFIC AREA}

For details of the proofs of the results presented in this section, and for further remarks and comments, we refer to Villa (2010).

Let us consider a Boolean model $\Xi$ in $\mathbb{R}^{d}$ with the notation introduced above. In order to provide sufficient conditions on $\Xi$ such that its specific area exists finite, it is intuitive by Eq. 7 and Theorem 6 why the following Assumptions implies Eq. 12 for $\sigma_{\Xi}(x)$ in Proposition 7.

In the assumption (A1) below the notation $\Theta \supseteq$ $\partial Z_{0}$ with $\mathbb{E}_{Q}\left[\mathscr{H}^{d-1}(\Theta)\right]<\infty$ means that for any $s \in$ $\mathbf{K}$ there exists a closed set $\Theta(s) \supseteq Z_{0}(s)$ such that $\int_{\mathbf{K}} \mathscr{H}^{d-1} \Theta(s) Q(\mathrm{~d} s)<\infty$.

Assumptions: (A1) $\partial Z_{0}$ is countably $\mathscr{H}^{d-1}$ rectifiable and compact, and such that there exist 
$\gamma>0$ and a random closed set $\Theta \supseteq \partial Z_{0}$ with $\mathbb{E}_{Q}\left[\mathscr{H}^{d-1}(\Theta)\right]<\infty$ such that, for $Q$-a.e. $s \in \mathbf{K}$,

$\mathscr{H}^{d-1}\left(\Theta(s) \cap B_{r}(x)\right) \geq \gamma r^{d-1} \forall x \in \partial Z_{0}(s), \forall r \in(0,1)$.

(A2) $\mathscr{H}^{d-1}(\operatorname{disc} f)=0$ and $f$ is locally bounded such that for any compact set $K \subset \mathbb{R}^{d}$

$$
\sup _{y \in K_{\oplus \operatorname{diam}\left(z_{0}\right)}} f(y) \leq \xi_{K},
$$

for some random variable $\xi_{K}$ with $\mathbb{E}_{Q}\left[\mathscr{H}^{d-1}(\Theta) \xi_{K}\right]<$ $\infty$.

Proposition 7 Let $\Xi$ be a Boolean model as in the Assumptions. Then

$$
\begin{aligned}
& \sigma_{\Xi}(x)=\exp \left\{-\mathbb{E}_{Q}\left[\int_{Z^{x}} f(y) \mathrm{d} y\right]\right\} \\
& \cdot \mathbb{E}_{Q}\left[\int_{\partial^{*} Z^{x}} f(y) \mathscr{H}^{d-1}(\mathrm{~d} y)\right. \\
&\left.+2 \int_{\partial Z^{x} \cap\left(Z^{x}\right)^{0}} f(y) \mathscr{H}^{d-1}(\mathrm{~d} y)\right],
\end{aligned}
$$

for all $x \in \mathbb{R}^{d}$.

Remark 8 The assumption (A1) is often fulfilled with $\Theta=\partial Z_{0}$ or $\Theta=\partial Z_{0} \cup \widetilde{A}$ for some sufficiently regular random closed set $\tilde{A}$. As a matter of fact, it can be seen as the stochastic version of Eq. 10, which, in many applications, is satisfied with $\eta(\cdot)=\mathscr{H}^{n}(\widetilde{A} \cap \cdot)$ for some closed set $\widetilde{A} \supseteq A$, as proved in (Ambrosio et al., 2000, p. 111) (see also Ambrosio et al., 2008).

The other integrability assumptions in (A1) and (A2) are just technical assumptions in order to exchange limit and integral in Eq. 7. We may notice that if $f$ is bounded, then $\xi_{K}$ is constant for any $K$, and so $\mathbb{E}_{Q}\left[\mathscr{H}^{d-1}(\Theta) \xi_{K}\right]$ is finite by (A1).

Having now an explicit formula for the specific area of inhomogeneous Boolean models as in the Assumptions, we may ask when $\sigma_{\Xi}$ coincides with $\lambda_{\partial \Xi}$, the mean surface density of $\Xi$. The next theorem shows that, without any further regularity assumption on $Z_{0}, \sigma_{\Xi}$ may differ form the mean surface density $\lambda_{\partial \Xi}$ of $\Xi$, in general.

Theorem 9 If $\Xi$ is a Boolean model as in the Assumptions satisfying Eq. 6, then

$$
\sigma_{\Xi}(x)=\lambda_{\partial * \Xi}(x)+2 \lambda_{\partial \Xi \cap \Xi^{0}}(x)
$$

for $\mathscr{H}^{d}$-a.e. $x \in \mathbb{R}^{d}$, where $\lambda_{\partial^{*} \Xi}$ and $\lambda_{\partial \Xi \cap \Xi^{0}}$ are the densities of $\mathbb{E}\left[\mathscr{H}_{\mid \partial^{*} \Xi}^{d-1}\right]$ and $E\left[\mathscr{H}_{\mid \partial \Xi \cap \Xi^{0}}^{d-1}\right]$, respectively.
Remark 10 By the proof of Theorem 9 it follows in particular that $\Xi$ admits local mean outer Minkowski content, i.e., for any Borel set $B \subset \mathbb{R}^{d}$ such that $\mathbb{E}\left[\mathscr{H}^{d-1}(\partial \Theta \cap \partial B)\right]=0$ it holds

$$
\begin{aligned}
& \lim _{r \downarrow 0} \frac{\mathbb{E}\left[\mathscr{H}^{d}\left(\left(\Xi_{\oplus r} \backslash \Xi\right) \cap B\right)\right]}{r} \\
&=\mathbb{E}\left[\mathscr{H}_{\mid \Xi^{1 / 2}}^{d-1}(B)\right]+2 \mathbb{E}\left[\mathscr{H}_{\mid \partial \Xi \cap \Xi^{0}}^{d-1}(B)\right] .
\end{aligned}
$$

We point out that the Assumptions, which guarantee the equation above, are quite general (see also Remark 8); the random sets which don't admit local mean outer Minkowski content could be considered as "pathological" sets.

Theorem 9 tells us that, in general, it may well happen that the specific area does not coincide with the mean surface density. We mentioned in the Introduction that a problem of interest in image analysis is the estimation of the mean surface density of random sets, and that the 2-D box approximations of points in $\mathbb{R}^{2}$ by pixels in computer graphics suggests the use of $\sigma_{\Xi}$ for the estimation of the mean surface density. Therefore we provide now sufficient conditions on $\Xi$ such that $\sigma_{\Xi}(x)=\lambda_{\partial \Xi}$ for $\mathscr{H}^{d}$ a.e. $x \in \mathbb{R}^{d}$. The Assumptions above on $\Xi$ imply that $\mathbb{E}\left[\mathscr{H}^{d-1}(\partial \Xi)\right]<\infty \mathbb{P}$-a.s., so that its mean surface density can be decomposed as follows:

$$
\lambda_{\partial \Xi}=\lambda_{\partial \Xi \cap \Xi^{0}}+\lambda_{\partial^{*} \Xi}+\lambda_{\partial \Xi \cap \Xi^{1}} .
$$

Then, it follows that $\sigma_{\Xi}(x)=\lambda_{\partial \Xi}(x)=\lambda_{\partial * \Xi}(x)$ for $\mathscr{H}^{d}$-a.e. $x \in \mathbb{R}^{d}$ if $\mathbb{E}\left[\mathscr{H}_{\mid \partial \Xi \cap\left(\Xi^{0} \cup \Xi^{1}\right)}^{d-1}\right]=0$.

The following proposition provides a sufficient regularity condition on the typical grain in order to have $\sigma_{\Xi}=\lambda_{\partial \Xi}$.

Proposition 11 Let $\Xi$ be a Boolean model as in the Assumptions satisfying Eq. 6, such that

$$
\mathbb{E}_{Q}\left[\mathscr{H}^{d-1}\left(\partial^{*} Z_{0}\right)\right]=\mathbb{E}_{Q}\left[\mathscr{H}^{d-1}\left(\partial Z_{0}\right)\right] .
$$

Then

$$
\begin{aligned}
\sigma_{\Xi}(x)= & \exp \left\{-\mathbb{E}_{Q}\left[\int_{Z^{x}} f(y) \mathrm{d} y\right]\right\} . \\
& \cdot \mathbb{E}_{Q}\left[\int_{\partial Z^{x}} f(y) \mathscr{H}^{d-1}(\mathrm{~d} y)\right]=\lambda_{\partial \Xi}(x)
\end{aligned}
$$

for $\mathscr{H}^{d}$-a.e. $x \in \mathbb{R}^{d}$. 
We point out that there exist (although a bit "pathological") subsets of $\mathbb{R}^{d}$ such that the $\mathscr{H}^{d-1}$. measure of their boundary differs from that one of their essential boundary ( $c f$. Ambrosio et al., 2008, Sect. 5); so, by choosing a set of this kind as deterministic typical grain, the condition Eq. 14 is not satisfied, and $\lambda_{\partial \Xi} \neq \sigma_{\Xi}$ for the corresponding Boolean model $\Xi$. Furthermore, it can be shown (Ambrosio et al., 2008) that the class of subsets $A$ of $\mathbb{R}^{d}$ such that $\mathscr{H}^{d-1}(\partial A)=\mathscr{H}^{d-1}\left(\partial^{*} A\right)$ is not stable under finite unions, that is, even if two subsets $A_{1}$ and $A_{2}$ of $\mathbb{R}^{d}$ are such that $\mathscr{H}^{d-1}\left(\partial A_{i}\right)=\mathscr{H}^{d-1}\left(\partial^{*} A_{i}\right), i=$ 1,2 , it may well happen that $\mathscr{H}^{d-1}\left(\partial\left(A_{1} \cup A_{2}\right)\right) \neq$ $\mathscr{H}^{d-1}\left(\partial^{*}\left(A_{1} \cup A_{2}\right)\right)$. Noticing that, by condition Eq. 6, $\Xi$ is almost surely a locally finite union of grains, Proposition 11 tells us that a sort of stability under finite unions for the expected value holds for Boolean models, because Eq. 14 implies that $\mathbb{E}\left[\mathscr{H}^{d-1}(\partial \Xi)\right]=$ $\mathbb{E}\left[\mathscr{H}^{d-1}\left(\partial^{*} \Xi\right)\right]$.

Since $\partial^{*} Z_{0} \subset \partial Z_{0}$, condition Eq. 14 is satisfied if $\mathscr{H}^{d-1}\left(\partial^{*} Z_{0}\right)=\mathscr{H}^{d-1}\left(\partial Z_{0}\right) \mathbb{P}$-a.s.; we remind that any compact subset $A$ of $\mathbb{R}^{d}$ with Lipschitz boundary satisfies $\mathscr{H}^{d-1}\left(\partial^{*} A\right)=\mathscr{H}^{d-1}(\partial A)$, and that the same holds also for a certain class of compact sets with positive reach, containing, in particular, all the $d$ dimensional convex bodies ( $c f$. Ambrosio et al., 2008; Villa, 2010). Therefore we may claim that $\sigma_{\Xi}=\lambda_{\partial \Xi}$ $\mathscr{H}^{d}$-a.e. for all Boolean models with typical grain $Z_{0}$ satisfying regularity conditions of this kind, and so, starting by the definition of $\sigma_{\Xi}$, estimators for the mean surface density $\lambda_{\partial \Xi}$ of $\Xi$ can be provided (see section below). We also mention that examples of applications of the above results to Boolean models of balls and segments are provided in Villa (2010, Sect. 5.3).

Remark 12 It is clear that Proposition 7 and Proposition 11 can be easily specified for the particular cases in which $\Xi$ is stationary (in such case $f$ is constant, say $c$, and so $\sigma_{\Xi}$ is constant as well, equal to $\left.e^{-c \mathbb{E}_{Q}\left[\mathscr{H}^{d}\left(Z_{0}\right)\right]} c \mathbb{E}_{Q}\left[\mathscr{S} \mathscr{M}\left(Z_{0}\right)\right]\right)$, or $\Xi$ has deterministic typical grain (in such case it is sufficient to assume that $Z_{0}$ is a compact set in $\mathscr{O}$, or $\mathscr{O}^{\prime}$, and that the intensity $f$ is locally bounded such that $\left.\mathscr{H}^{d-1}(\operatorname{disc} f)=0\right)$.

Let us also notice that $\sigma_{\Xi}(x)$ might be not trivial for $(d-1)$-dimensional Boolean models, as well; it can be shown that under similar assumptions to (A1) and (A2),

$$
\begin{aligned}
\sigma_{\Xi}(x) & =2 \lambda_{\partial \Xi}(x)=\mathbb{E}_{Q}\left[\int_{Z^{x}} f(y) \mathscr{H}^{d-1}(\mathrm{~d} y)\right], \\
\mathscr{H}^{d} \text {-a.e. } x & \in \mathbb{R}^{d} .
\end{aligned}
$$

We refer to Villa (2010, Sect. 5) (see also Villa, 2009b) for a more detailed discussion of these particular cases.

\section{SOME APPLICATIONS}

In this section we shall discuss a couple of applications of the results above; in particular, applications to birth-and-growth processes which are of interest in several application areas as Material Science, Biology, etc.

\section{An estimator of the mean surface density}

It could be of interest in Image Analysis to estimate the mean surface density of random closed sets by means of their Minkowski enlargements, because of the computer graphics representation of lower dimensional sets in terms of pixels. By repeating the same argument in Villa (2010, Prop. 6.1), where estimators for the mean density of lower dimensional random closed sets are provided, in Villa (2009b) a natural estimator for $\lambda_{\partial \Xi}$ has been introduced, being $\Xi$ a Boolean model as in the assumptions of Proposition 11, and so

$$
\lambda_{\partial \Xi}(x)=\lim _{r \downarrow 0} \frac{\mathbb{P}\left(x \in \Xi_{\oplus r} \backslash \Xi\right)}{r},
$$

for $\mathscr{H}^{d}$-a.e. $x \in \mathbb{R}^{d}$.

Given an i.i.d. random sample $\Xi_{1}, \ldots, \Xi_{N}$ of $\Xi$, the equation above suggests the following estimator of $\lambda_{\partial \Xi}(x)$ :

$$
\begin{aligned}
\hat{\lambda}_{\partial \Xi}^{N}(x):=\frac{\sum_{i=1}^{N} \mathbf{1}_{\Xi_{i_{\oplus R_{N}}} \backslash \Xi_{i}}(x)}{N R_{N}} \\
=\frac{\sum_{i=1}^{N}\left(\mathbf{1}_{\Xi_{i} \cap B_{R_{N}}(x) \neq \emptyset}-\mathbf{1}_{\Xi_{i} \cap\{x\} \neq \emptyset}\right)}{N R_{N}},
\end{aligned}
$$

with $R_{N}$ such that

$$
\lim _{N \rightarrow \infty} R_{N}=0 \quad \text { and } \quad \lim _{N \rightarrow \infty} N R_{N}=\infty .
$$

It is not difficult to check that $\widehat{\lambda}_{\partial \Xi}^{N}(x)$ is an asymptotically unbiased and consistent estimator of $\lambda_{\partial \Xi}(x)$ for $\mathscr{H}^{d}$-a.e. $x \in \mathbb{R}^{d}$.

Remark 13 We also mention that the outer Minkowski content concept as measure of the boundary of deterministic sets, has been recently used to provide estimators for the $\mathscr{H}^{d-1}$-measure of the boundary of compact subsets of $\mathbb{R}^{d}$ in Armendáriz et al. (2009). Therefore, open problems related to the estimation of the mean boundary measure of random closed sets might be the following: the study of the statistical properties of the estimator above, and the generalization to random closed sets of the results in Armendáriz et al. (2009). 


\section{Applications to birth-and-growth processes}

Many real phenomena as crystallization processes (Vandermeer et al., 1991; Capasso, 2003, and references therein), tumor growth (Anderson, 2003), etc., may be modelled as evolving random closed sets, that is as full dimensional time dependent random closed sets. In particular, any real situation in which nuclei are born in time and are located in space randomly, and each nucleus generates a grain evolving in time according to a given growth law, may be modelled as space-time structured stochastic birthand-growth processes. Such a process is described by a marked point process, say $N:=\left\{\left(T_{j}, X_{j}\right)\right\}_{j \in \mathbb{N}}$, modelling births at random times $T_{j} \in \mathbb{R}_{+}$and related random spatial locations (nuclei) $X_{j} \in \mathbb{R}^{d}(d \geq 2)$, and by a growth model.

Denoting by $\Theta_{t_{0}}^{t}\left(x_{0}\right)$ the grain born at some time $t_{0}$ and location $x_{0}$, and grown up to time $t$, under regularity assumptions on the birth and growth model, the union set

$$
\Theta^{t}=\bigcup_{n: T_{n} \leq t} \Theta_{T_{n}}^{t}\left(X_{n}\right)
$$

of such grains at time $t$ is then a locally finite union of random closed sets. The family $\left\{\Theta^{t}\right\}_{t}$ is called birthand-growth process, and a problem of interest in many applications is to find evolution equations for the mean volume density, in terms of the mean surface density of $\Theta^{t}$.

A lot of papers on this subject can be found in literature; in particular, the case in which $N$ is given by a marked Poisson process has been studied extensively (Kolmogorov, 1937; Burger et al., 2006, and references therein).

In this section we want to point out the role of the existence of the (local) mean outer Minkowski content and the specific area of $\Theta^{t}$ in the study of the mean volume and surface densities of $\Theta^{t}$. We recall that the mean volume density of $\Theta^{t}$, usually denoted by $V_{V}(t, \cdot)$, is the Radon-Nikodym derivative of the measure $\mathbb{E}\left[\mathscr{H}_{\mid \Theta^{t}}^{d}\right]$, i.e.,

$$
\mathbb{E}\left[\mathscr{H}^{d}\left(\Theta^{t} \cap B\right)\right]=\int_{B} V_{V}(t, x) \mathrm{d} x, \quad \forall B \in \mathscr{B}_{\mathbb{R}^{d}} ;
$$

similarly, the mean surface density of $\Theta^{t}$, usually denoted by $S_{V}(t, \cdot)$, is the Radon-Nikodym derivative of the measure $\mathbb{E}\left[\mathscr{H}_{\mid \partial \Theta^{d}}^{d-1}\right]$, i.e.,

$$
\mathbb{E}\left[\mathscr{H}^{d-1}\left(\partial \Theta^{t} \cap B\right)\right]=\int_{B} S_{V}(t, x) \mathrm{d} x, \quad \forall B \in \mathscr{B}_{\mathbb{R}^{d}} .
$$

Let us notice that

$$
V_{V}(t, x)=\mathbb{P}\left(x \in \Theta^{t}\right),
$$

whereas $S_{V}(t, x)=\lambda_{\partial \Theta^{t}}(x)$, according to our previous notation.

We assume here that the nucleation process $N$ is a Poisson marked point process (equivalently, a Poisson point process in $\mathbb{R}_{+} \times \mathbb{R}^{d}$ ), with intensity measure $\Lambda$ of the type

$$
\Lambda(\mathrm{d}(t, x))=\alpha(t, x) \mathrm{d} t \mathrm{~d} x
$$

and satisfying the usual condition (Eq. 6).

Models of volume growth have been studied extensively, since the pioneering work by Kolmogorov (1937). Clearly, different kinds of growth model give rise to different kinds of processes $\left\{\Theta^{t}\right\}_{t}$.

Let us first consider the normal growth model (Capasso and Villa, 2007, and references therein), according to which, at $\mathscr{H}^{d-1}$-almost every point of the actual grain surface at time $t$ (i.e., at $\mathscr{H}^{d-1}$-a.e. $\left.x \in \partial \Theta_{T_{j}}^{t}\left(X_{j}\right)\right)$, growth occurs with a given strictly positive normal velocity

$$
v(t, x)=G(t, x) n(t, x),
$$

where $G(t, x)$ is a given deterministic growth field, and $n(t, x)$ is the unit outer normal at point $x \in \partial \Theta_{T_{0}}^{t}\left(X_{0}\right)$. We assume that

$$
0<g_{0} \leq G(t, x) \leq G_{0}<\infty \quad \forall(t, x) \in \mathbb{R}_{+} \times \mathbb{R}^{d},
$$

for some $g_{0}, G_{0} \in \mathbb{R}$, and that $G(t, x)$ is sufficiently regular such that the evolution problem given by (16) for the growth front $\partial \Theta_{t_{0}}^{t}(x)$ is well posed. It follows that for any fixed $t \in \mathbb{R}_{+}$, the topological boundary of each grain is a random closed set with locally finite $\mathscr{H}^{d-1}$-measure $\mathbb{P}$-almost surely (Burger, 2002). This and the assumption that $\Theta^{t}$ is a locally finite union of grains imply that $\mathbb{P}$-almost surely $\mathscr{H}^{d-1}\left(\partial \Theta^{t}\right)<$ $\infty$, and so that $\Theta^{t}$ has density 1 or $1 / 2$ at $\mathscr{H}^{d-1}$ a.e. point of its boundary $\mathbb{P}$-almost surely. We can claim the assumptions on the growth model imply that $\mathscr{H}^{d-1}\left(\left(\Theta^{t}\right)^{0}\right)=0 \mathbb{P}$-a.s.

For the normal growth model above with Poissonian nucleation process, recent results (Capasso and Villa, 2008, Prop. 25; Capasso and Villa, 2007, Prop. 19; Villa, 2008, Prop. 2.3) show that, if $\Theta^{t}$ admits local mean outer Minkowski content for all $t>0$ and if the random variable $T(x)$ defined by

$$
T(x):=\min \left\{t>0: x \in \Theta^{t}\right\}
$$

is continuous with density, then the following evolution equation holds in weak form:

$$
\frac{\partial}{\partial t} V_{V}(t, x)=G(t, x) S_{V}(t, x) .
$$


The random variable $T(x)$ is called time of capture of point $x$, and its probability density function, say $p_{T(x)}$, is just the partial derivative of $V_{V}$ with respect to $t$ (Capasso and Villa, 2008, Eq. 14). By repeating the same arguments of Proposition 25 in Capasso and Villa (2008) and assuming that $\Theta^{t}$ admits local mean outer Minkowski content, we can state the following slightly more general result. Note that the assumption that the boundary of $\Theta^{t}$ is sufficiently regular so that $\Theta^{t}$ admits local mean outer Minkowski content, is not so restrictive for applications (see Remark 10).

Proposition 14 Let $\left\{\Theta^{t}\right\}_{t}$ be a birth-and-growth process with normal growth model as above, and sufficiently regular such that $\Theta^{t}$ admits local mean outer Minkowski content Eq. 13. Then the following equation holds in weak form

$$
\frac{\partial}{\partial t} V_{V}(t, x)=G(t, x) \lambda_{\partial^{*} \Theta^{t}}(x) .
$$

\section{Proof. (Sketch)}

The assumptions on the nucleation process and on the growth model imply (Villa, 2008, Theorem 3.3) that $T(x)$ admits probability density function $p_{T(x)}$. By Eqs. 17 and 24 in Capasso and Villa (2008), the following chain of equalities hold

$$
\begin{aligned}
& \frac{\partial}{\partial t} V_{V}(t, x)=p_{T(x)}=h(t, x) \mathbb{P}\left(x \notin \Theta^{t}\right) \\
& =G(t, x) \frac{\partial}{\partial r} \mathbb{P}\left(x \in \Theta_{\oplus r}^{t} \backslash \Theta^{t}\right)_{\mid r=0}=G(t, x) \sigma_{\Theta^{t}}(x),
\end{aligned}
$$

where $h(t, x)$ is the so-called hazard function associated to the point $x$ at time $t$ (Capasso and Villa, 2008, Def. 21). Eq. 13 can be written equivalently (in weak form) as

$$
\begin{aligned}
\frac{\partial}{\partial r} \mathbb{P}(x & \left.=\Theta_{\oplus r}^{t} \backslash \Theta^{t}\right)_{\mid r=0} \\
& =\lambda_{\partial^{*} \Theta^{t}}(x)+2 \lambda_{\partial \Theta^{t} \cap\left(\Theta^{t}\right)^{0}}(x)=\lambda_{\partial^{*} \Theta^{t}}(x),
\end{aligned}
$$

where the last equation follows by having observed that $\mathscr{H}^{d-1}\left(\left(\Theta^{t}\right)^{0}\right)=0 \mathbb{P}$-a.s., and so we obtain Eq. 18.

Remark 15 Let us notice that the following decomposition of the mean surface density holds

$$
\lambda_{\partial \Theta^{t}}=\lambda_{\partial * \Theta^{t}}+\lambda_{\partial \Theta^{t} \cap\left(\Theta^{t}\right)^{0}}+\lambda_{\partial \Theta^{t} \cap\left(\Theta^{t}\right)^{1}}
$$

As a consequence, since $\lambda_{\partial \Theta^{t} \cap\left(\Theta^{t}\right)^{0}}=0$, Eq. 18 can be equivalently written

$$
\frac{\partial}{\partial t} V_{V}(t, x)=G(t, x)\left(\lambda_{\partial \Theta^{t}}(x)-\lambda_{\partial \Theta^{t} \cap\left(\Theta^{t}\right)^{1}}\right) ;
$$

assuming now that for a.e. $t \in \mathbb{R}_{+} \mathbb{E}\left[\mathscr{H}_{\mid \partial \Theta^{t} \cap\left(\partial \Theta^{t}\right)^{1}}^{d-1}\right]=0$ , then for a.e. $t \in \mathbb{R}_{+} \lambda_{\partial^{*} \Theta^{t}}=\lambda_{\partial \Theta^{t}}$ and Eq. 18 coincides with Eq. 17.

Let us now consider a different growth model. We assume now that each grain grows with the same growth law of a "typical grain" with its nucleus at the origin; in other words, the grain $\Theta_{s}^{t}(x)$ born at point $x$ at time $s$ and grown up to time $t$ can be seen as the translation in $x$ of a grain born in 0 at the same birth-time. By using the notation introduced for Boolean models, given the nucleation process $\left\{\left(T_{n}, X_{n}\right)\right\}_{n}$, where now the marks are the birth times $T_{n}$ associated to the spatial locations $X_{n}$ of the nuclei, we can model the crystallized region $\Theta^{t}$ at time $t$ as the Boolean model

$$
\Theta^{t}=\bigcup_{\left(T_{n}, X_{n}\right) \in N: T_{n} \leq t} X_{n}+Z_{0}\left(T_{n}\right)=X_{n}+\Theta_{T_{n}}^{t}(0) .
$$

Notice that such a model may be used to describe real situations in which the growth model is assumed to be such that the shape of the grains is preserved during the process (e.g., prolates and spheroids in Vandermeer et al., 1991). Clearly, the particular case of spherical growth (which corresponds to the previous growth model with $G=G(t)$ ), can be described also by means of the present model with $Z_{0}\left(T_{n}\right)=B_{R\left(T_{n}, t\right)}(0)$, where $R\left(T_{n}, t\right)=\int_{T_{n}}^{t} G(s) \mathrm{d} s$.

Thus, if the Boolean model $\Theta^{t}$ satisfies the Assumptions, then by Proposition 7 it follows that the specific area $\sigma_{\Theta^{t}}$ exists given by Eq. 12; if furthermore condition Eq. 14 is satisfied (usually true in many real applications), then an explicit formula for the mean surface density (equivalently $S_{V}$ ) can be obtained by Eq. 15. In order to apply such formula to the birthand-growth process in Eq. 19, note that the mark distribution $Q$ represents now the probability law of the birth time of the typical grain; for instance, if the nucleation rate is constant in time, then, for any fixed $t$, $Q$ is the uniform distribution in $[0, t]$. More in general, for any fixed $t$, since the nucleation takes place during the time interval $[0, t]$, Eq. 15 will be of the type

$$
\begin{array}{r}
\sigma_{\Theta^{t}}(x)=S_{V}(t, x)=\exp \left\{-\int_{0}^{t} \int_{x-\Theta_{s}^{t}(0)} f(y) \mathrm{d} y Q \mathrm{~d} s\right\} . \\
\cdot \int_{0}^{t} \int_{\partial\left(x-\Theta_{S}^{t}(0)\right)} f(y) \mathscr{H}^{d-1}(\mathrm{y}) Q(\mathrm{~d} s) .
\end{array}
$$

Remark 16 Whenever the nucleation is homogeneous in space, then $f$ is constant and the above formula 
simplify as follows

$$
\begin{aligned}
S_{V}(t, x)=\exp \left\{-\int_{0}^{t} c \mathscr{H}^{d}\left(\Theta_{s}^{t}(0)\right) Q \mathrm{~d} s\right\} . \\
\cdot \int_{0}^{t} \mathscr{H}^{d-1}\left(\partial\left(\Theta_{s}^{t}(0)\right) Q(\mathrm{~d} s),\right.
\end{aligned}
$$

in accordance with Vandermeer et al. (1991, Eqs. 912)

\section{ACKNOWLEDGEMENTS}

I wish to thank the anonymous Referees for their careful reading, and for their useful comments which improved significantly the quality of this paper. A preliminary version appeared in Villa (2009b).

\section{REFERENCES}

Ambrosio L, Capasso V, Villa E (2009). On the approximation of mean densities of random closed sets. Bernoulli 15:1222-42.

Ambrosio L, Colesanti A, Villa E (2008). Outer Minkowski content for some classes of closed sets. Math Ann 342:727-48.

Ambrosio L, Fusco N, Pallara D (2000). Functions of bounded variation and free discontinuity problems. Oxford: Clarendon Press.

Anderson ARA (2003). Effects of cell adhesion on solid tumour geometry. In: Sekimura $\mathrm{T}$ et al.eds. Morphogenesis and Pattern Formation in Biological Systems. Tokyo: Springer-Verlag.

Armendáriz I, Cuevas A, Fraiman R (2009). Nonparametric estimation of boundary measures and related functionals: asymptotic results. Adv Appl Prob 41:311-22.

Baddeley A, Barany I, Schneider R, Weil W (2007). Stochastic Geometry. Lect Not Math 1982. Berlin: Springer.

Burger M (2002). Growth fronts of first-order HamiltonJacobi equations. SFB Report 02-8. Linz: J. Kepler University.

Burger M, Capasso V, Pizzocchero L. (2006). Mesoscale averaging of nucleation and growth models. SIAM J Multiscale Model Sim 5:564-92.

Capasso V (2003). Mathematical modelling for polymer processing. Polymerization, crystallization, manufacturing. Mathematics in Industry Vol 2. Heidelberg: Springer Verlag.

Capasso V, Villa E (2007). On mean densities of inhomogeneous geometric processes arising in material science and medicine. Image Anal Stereol 26:23-36.

Capasso V, Villa E (2008). On the geometric densities of random closed sets. Stoch Anal Appl 26:784-808.
Falconer KJ (1985). The geometry of fractal sets. Cambridge: Cambridge University Press.

Federer H (1969). Geometric measure theory. Berlin: Spriger.

Hug D (2000). Contact distributions of Boolean models. Rend Circ Mat Palermo 2 Suppl 65:137-81.

Hug D, Last G (2000). On support measures in Minkowski spaces and contact distributions in stochastic geometry. Ann Prob 28:796-850.

Hug D, Last G, Weil W (2004). A local Steiner-type formula for general closed sets and applications Math Z 246:237-72.

Hug D, Last G, Weil W (2006). Polynomial parallel volume, convexity and contact distributions of random sets. Probab Theory Rel 135:169-200.

Kärkkäinen S, Jensen EBV, Jeulin D (2002). On the orientational analysis of planar fibre systems from digital images. J Microsc 207:69-77.

Kiderlen M, Rataj J (2006). On infinitesimal increase of volumes of morphological transforms. Mathematika 53:103-27.

Kolmogorov AN (1937). On the statistical theory of the crystallization of metals. Bull Acad Sci USSR Math Ser $1: 355-9$.

Matheron G (1975). Random sets and integral geometry. New York: John Wiley \& Sons.

Schneider R, Weil W (2008). Stochastic and integral geometry. Berlin, Heidelberg: Springer.

Stoyan D, Kendall WS, Mecke J (1995). Stochastic geometry and its applications. Chichester: Wiley.

Vandermeer RA, Masumura RA, Rath BB (1991). Microstructural paths of shape-preserved nucleation and growth transformations. Acta Metall Mater 39:383-9.

Villa E (2008) A note on mean volume and surface densities for a class of birth-and-growth stochastic processes. Int J Contemp Math Sci 3:1141-55

Villa E (2009a). On the outer Minkowski content of sets. Ann Mat Pura Appl 188:619-30.

Villa E (2009b) Specific area and mean surface density of inhomogeneous Boolean models. In: Stereology and Image Analysis. ECS10: Proceeding of the 10th European Conference of ISS, (V.Capasso et al. Ed.), The MIRIAM Project Series, Vol. 4, Esculapio, Bologna, 485-90.

Villa E (2010). Mean densities and spherical contact distribution function of inhomogeneous Boolean models. Stoch Anal Appl 28:480-504.

Weil W. (2001). Densities of mixed volumes for Boolean models. Adv Appl Prob 33:39-60. 